\title{
Erratum
}

\section{Technetium and lithium in Galactic bulge AGB stars}

\author{
S. Uttenthaler ${ }^{1}$, J. Hron ${ }^{1}$, T. Lebzelter ${ }^{1}$, M. Busso ${ }^{2}$, S. Palmerini ${ }^{2}$, \\ M. Schultheis ${ }^{3}$, H. U. Käuff ${ }^{4}$, M. T. Lederer ${ }^{1}$, and B. Aringer ${ }^{1}$ \\ 1 Institute for Astronomy, University of Vienna, Türkenschanzstraße 17, 1180 Vienna, Austria \\ e-mail: [uttenthaler; hron; lebzelter; lederer; aringer]@astro.univie.ac.at \\ 2 Department of Physics, University of Perugia, via A. Pascoli 1, 06123 Perugia, Italy \\ e-mail: [busso;palmerini]@fisica.unipg.it \\ 3 Observatoire de Besançon, 41bis, avenue de l'Observatoire, BP 1615, 25010 Besançon Cedex, France \\ e-mail: mathias@obs-besancon.fr \\ 4 European Southern Observatory, Karl Schwarzschild Straße 2, 85748 Garching near Munich, Germany \\ e-mail: hukaufl@eso.org \\ A\&A 463, 251-259 (2007), DOI: 10.1051/0004-6361:20065463 \\ A\&A 471, L41-L45 (2007), DOI: 10.1051/0004-6361:20077879
}

Key words. stars: late-type - stars: AGB and post-AGB - stars: evolution - errata, addenda

The near-IR photometry of bulge AGB variables analysed in Uttenthaler et al. (2007a) and Uttenthaler et al. (2007b) was de-reddened incorrectly, because of erroneous galactic latitudes used. Table 1 reproduces the corrected mean $J_{0}$ and $K_{0}$ magnitudes of the stars.

When using the thus corrected values, one star (M 1313) appears as strong outlier in the CMD with a $(J-K)_{0}$ colour of 1.61. For this star, ESO near-IR photometry has been acquired at two epochs (Schultheis et al. 1998). Using the ESO photometry only, M 1313 would have $(J-K)_{0}=1.71$. We suspect that the ESO photometry of M 1313 is in error, too. We thus quote the $J_{0}$ and $K_{0}$ magnitudes based on the 2MASS measurement only, which yields $(J-K)_{0}=1.47$.

We want to note that five out of 27 spectroscopically confirmed oxygen-rich sample stars have a $(J-K)_{0}$ colour redder than the commonly used limit between oxygen-rich M-type and carbon-rich C-type stars of 1.4 (Battinelli et al. 2007).

The main conclusions drawn in Uttenthaler et al. (2007a) and Uttenthaler et al. (2007b) remain unaltered. The bolometric magnitudes of the Tc-rich as well as of the Li-rich stars identified in these papers are altered by only a few 0.01 at most. The biggest consequence is that several sample stars turn out to be bluer in $(J-K)_{0}$ after this correction, and the isochrone with $5 \mathrm{Gyr}$ age and solar metallicity of Girardi et al. (2000) now well describes the observed colour range when using our $(J-K)_{0}$ vs. $T_{\text {eff }}$ calibration (see Schultheis et al. 1998). Nevertheless, the observed AGB tip luminosity is higher than that of the isochrones from Girardi et al. (2000).

\section{References}

Battinelli, P., Demers, S., \& Mannucci, F. 2007, A\&A, 474, 35 Girardi, L., Bressan, A., Bertelli, G., \& Chiosi, C. 2000, A\&AS, 141, 371 Schultheis, M., Ng, Y. K., Hron, J., \& Kerschbaum, F. 1998, A\&A, 338, 581
Table 1. Corrected $J_{0}$ and $K_{0}$ values of the sample stars. The bolometric magnitude $M_{\mathrm{bol}}$ of the stars is corrected for the bulge depth scatter (see Uttenthaler et al. 2007a, for details)

\begin{tabular}{lccc}
\hline \hline Name & $J_{0}[\mathrm{mag}]$ & $K_{0}[\mathrm{mag}]$ & $M_{\text {bol }}$ \\
\hline M 45 & 8.10 & 6.65 & -4.53 \\
M 100 & 7.66 & 6.37 & -4.77 \\
M 143 & 9.21 & 7.96 & -4.03 \\
M 195 & 8.79 & 7.58 & -4.16 \\
M 277 & 8.43 & 7.11 & -4.51 \\
M 315 & 7.91 & 6.54 & -4.92 \\
M 331 & 8.06 & 6.60 & -4.80 \\
M 626 & 8.45 & 7.19 & -4.78 \\
M 794 & 7.39 & 6.04 & -4.78 \\
M 1147 & 7.27 & 5.77 & -5.27 \\
M 1179 & 8.56 & 7.20 & -4.58 \\
M 1287 & 7.95 & 6.64 & -4.85 \\
M 1313 & 7.71 & 6.24 & -5.19 \\
M 1347 & 7.47 & 5.99 & -5.43 \\
S70 & 8.44 & 7.15 & -3.60 \\
S328 & 8.95 & 7.75 & -3.58 \\
S639 & 8.71 & 7.45 & -3.62 \\
S719 & 7.91 & 6.61 & -4.64 \\
S942 & 7.89 & 6.55 & -5.00 \\
S1002 & 8.30 & 7.08 & -3.94 \\
S1008 & 7.97 & 6.72 & -4.28 \\
S1059 & 8.92 & 7.69 & -3.34 \\
S1176 & 8.27 & 6.94 & -3.79 \\
S1204 & 8.64 & 7.31 & -3.92 \\
S1470 & 8.55 & 7.34 & -3.84 \\
S1517 & 8.94 & 7.68 & -3.86 \\
S1991 & 9.17 & 8.02 & -3.09 \\
\hline & & &
\end{tabular}

Uttenthaler, S., Hron, J., Lebzelter, T., et al. 2007a, A\&A, 463, 251, Tc-paper Uttenthaler, S., Lebzelter, T., Palmerini, S., et al. 2007b, A\&A, 471, L41, Li-paper 